\title{
PREPARATION AND IN-VITRO EVALUATION OF EUDISPERT HYDROGEL RECTAL SUPPOSITORIES CONTAINING SALICYLAMIDE
}

\author{
Afaf A. Ramadan, Amal S. Abou El- Eineen and Dalia A. Attia
}

Department of Pharmaceutics and Industrial Pharmacy, Faculty of Pharmacy, Al-Azhar University, Nasr City, Cairo, Egypt

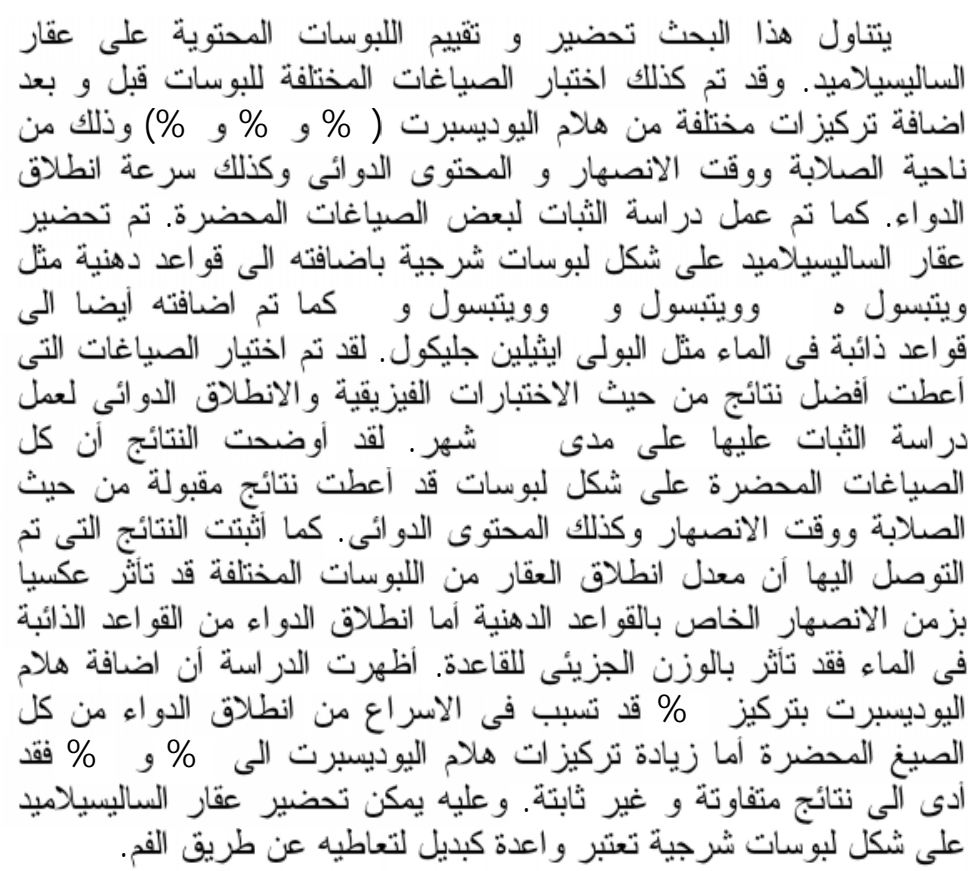

This article aimed to formulate and evaluate salicylamide suppositories. The prepared formulae with and without different concentrations of gels were tested for hardness, melting time, content uniformity and drug release. The stability of some of the selected formulae was assessed. Salicylamide was formulated as a rectal suppository with emulsifying fatty bases (witepsol H15, W25 and w35) and water - soluble bases (PEG) adopting the molding from a melt technique. Physical characteristics and dissolution profiles of the prepared formulations were determined. The effects 
of adding gel (eudispert hv) in concentrations of (2, 4 and 6\%) were also investigated.

Formulations showing high rank order were scaled up for shelflife stability study for one year. The results showed that all the investigated formulae have acceptable physical characteristics with respect to hardness, melting time and uniformity of drug content. The amount of drug released during $120 \mathrm{~min}$. was inversely affected by the melting point of the fatty base. The release from PEG bases was found to be molecular weight dependent. Addition of $2 \%$ eudispert gel increased the release from all the investigated formulae. Increasing gel concentration to $4 \%$ then to $6 \%$ showed different effects on the release. Rectal suppository of salicylamide could be prepared as an alternative to the oral dosage form to circumvent the first - pass metabolism ${ }^{l}$

\section{INTRODUCTION}

Oral administration is the route of choice in the daily practice of pharmacotherapy. However, in circumstances such as nausea, vomiting and unconsciousness, this becomes impractical. In these cases the rectal route may represent a practical alternative. This route is also useful when the patient is very young, very old, or mentally disturbed ${ }^{2}$. For drugs irritating the stomach $\mathrm{pH}$ or enzymes, the rectal route becomes an alternative $^{3 \& 4}$. Other reasons for preferring the rectal route over the oral one in drug administration is when the drug is extensively metabolized or deactivated by liver enzymes ${ }^{5}$. The human rectum represents a body cavity in which drugs can be easily introduced and retained with fairly good absorption, where, rectal therapy overcomes problems encountered with oral therapy such as first pass effect. On the other hand, the middle and inferior rectal veins drain the lower part of the rectum and venous blood is returned to the inferior vena cava. Therefore, drug absorbed in the latter system will be delivered preferentially to the systemic circulation by passing the liver and avoiding the first - pass metabolism ${ }^{6}$. Another mechanism enabling a drug to circumvent hepatic first - pass elimination is absorption into the lymphatic system ${ }^{7}$. However, the total amount of drug absorbed lymphatically appears to be quite small, possibly because of the low lymph flow compared to that of blood.

When a drug is administered as a suppository, several factors may influence the extent and rate of drug absorption into the general circulation $^{8}$. Melting or liquefaction of the fatty or hydrophilic suppository precedes release of the drug; the latter process is dependent on rectal environment, drug substance and 
suppository base. Rectal administration of some high clearance drugs such as propranolol and salicylamide did not result in higher bioavailability as compared with oral administration $^{9 \& 10}$.

Salicylamide, a mild analgesic and antipyretic agent, is used as a rubefacient in deep heating liniments. It has limited clinical effectiveness because of its extensive biotransformation after oral administration. However, it is quite valuable in the basic exploration of both the relationship between biotransformation and route of administration and the means by which pharmaceutical technology can produce drug preparations that avoid first - pass elimination $^{11-15}$.

Hydrogels are three dimensional polymer networks that swell but do not dissolve in aqueous media. They are used to regulate drug release in reservoir - based systems or as carriers in swelling - controlled release devices. The effect of gel on the release and absorption of drugs from rectal dosage forms was extensively studied ${ }^{16 \& 17}$. These rectal gel preparations showed excellent staying and bioadhesive effects in the lower part of the rectum in rats and rabbits $^{18-20}$.

\section{EXPERIMENTAL Materials}

Salicylamide was received as a gift from CID company. Witepsol $\mathrm{H}$ 15, Witepsol W 25 and Wiepsol W 35 were supplied by Nobel Dynamitte, Germany. Polyethylene glycol (PEG) 6000, 4000, 1540 and 1000 were supplied by BDH chemicals, LTD, Poole, England Eudispert; Higuchi Co., Tokoyo, Japan. Other materials were of analytical grade and used without further purification.

\section{Preparation of salicylamide suppositories}

Suppositories of 1 gram weight, each containing $100 \mathrm{mg}$ of salicylamide were prepared. The procedures briefly include melting different oleophilic bases (witepsol H15, W25 and W35) at their appropriate temperatures then incorporating salicylamide using electric stirrer until a homogenous mixture was formed. The mixture was poured into 1 gram capacity molds, allowing it to cool and congeal into suppositories. The suppositories were removed from the molds, stored in light - resistant containers and kept in a refrigerator.

\section{Preparation of gel containing suppositories}

Gels are prepared from eudispert hv with different concentrations $1 \%$, $2 \%$ and $3 \%$. Eudispert $1 \%$ prepared by suspending 1 gram in $100 \mathrm{ml}$ of boiling purified water and vortex then stirred mechanically at high speed. The mixture is being allowed to soak for about 10 - 20 minutes. Different ratios of the gels are mixed with suppository bases to achieve concentrations of 2, 4 and $6 \% \mathrm{w} / \mathrm{w}$ of each base. The medicated and unmedicated suppositories containing and not containing gels were allowed to settle for 2-3 days before testing. Composition of different formulations 
of salicylamide suppositories are shown in Table (1).

\section{Evaluation of salicylamide suppositories \\ Hardness test}

This test was carried out to measure the brittleness and fragility of suppositories. Hardness was determined at room temperature using Erweka suppository hardness tester. The mean hardness \pm standard deviation (SD) are presented in Table (2).

\section{Melting time}

This test is called softening time or macromelting range test and it is a measure of time for the entire suppository to melt or disperse when immersed in a constant - temperature $\left(37^{\circ} \mathrm{C}\right)$ water bath. The test is carried out on medicated suppository bases using Erweka apparatus, where one suppository was placed in each tube, the beaker was filled with $250 \mathrm{ml}$ phosphate buffer ( $\mathrm{pH}$ 7.4). the mean melting time \pm SD are presented in Table (2).

\section{Content uniformity}

Five suppositories were randomly chosen, weighed and allowed to dissolve or melt in $50 \mathrm{ml}$ distilled water and warmed to $40^{\circ} \mathrm{C}$. The whole solution was shaken and set aside at room temperature for one hour to attain an adequate separation. A sample of $10 \mathrm{ml}$ was withdrawn, filtered using Millipore filter, diluted and assayed spectrophotometrically at $302 \mathrm{~nm}^{21}$. The mean percent drug concentrations \pm SD were calculated and presented in Table (2).

Table 1: Composition of different formulations of salicylamide suppositories.

\begin{tabular}{||c|c|c|c||}
\hline $\begin{array}{c}\text { Formula } \\
\text { No. }\end{array}$ & $\begin{array}{c}\text { Composition of } \\
\text { suppository base }\end{array}$ & Melting point & Hydroxyl value \\
\hline F1 & Witepsol H15 & $33.5-35.5$ & $5-15$ \\
\hline F2 & Witepsol W25 & $33.5-35.5$ & $20-30$ \\
\hline F3 & Witepsol W35 & $33.5-35.5$ & $40-50$ \\
\hline F4 & $\begin{array}{c}\text { PEG 1540:PEG 6000 } \\
(70 \%: 30 \%)\end{array}$ & ----- & ----- \\
\hline F5 & $\begin{array}{c}\text { PEG 1540:PEG } \\
6000: \mathrm{H}_{2} \mathrm{O} \\
(30 \%: 50 \%: 20 \%)\end{array}$ & ----- & ----- \\
& $\begin{array}{l}\text { PEG } 1000: \text { PEG } \\
4000(75 \%: 25 \%)\end{array}$ & ----- & ---- \\
\hline F6 & \multicolumn{2}{|l}{} \\
\hline
\end{tabular}


Table 2: Physical characteristics of salicylamide suppositories.

\begin{tabular}{||c|c|c|c||}
\hline $\begin{array}{c}\text { Formula } \\
\text { No. }\end{array}$ & $\begin{array}{c}\text { Hardness (kg) } \\
\text { mean } \pm \text { SD }\end{array}$ & $\begin{array}{c}\text { Melting time(min.) } \\
\text { mean } \pm \text { SD }\end{array}$ & $\begin{array}{c}\text { Drug content }(\%) \\
\text { mean } \pm \text { SD }\end{array}$ \\
\hline F1 & $6.10 \pm 0.14$ & $21 \pm 1.0$ & $99.12 \pm 2.4$ \\
\hline F2 & $6.30 \pm 0.2$ & $19 \pm 0.5$ & $98.44 \pm 3.1$ \\
\hline F3 & $6.25 \pm 0.09$ & $25 \pm 1.0$ & $101.30 \pm 1.9$ \\
\hline F4 & $6.00 \pm 43$ & $22 \pm 1.0$ & $100.21 \pm 3.4$ \\
\hline F5 & $6.45 \pm 22$ & $35 \pm 1.5$ & $102.13 \pm 2.2$ \\
\hline F6 & $5.80 \pm 11$ & $25.5 \pm 0.5$ & $99.81 \pm 2.5$ \\
\hline
\end{tabular}

\section{In-vitro release studies}

The USP rotating basket dissolution apparatus was used for the determination of release rates of salicylamide from different suppository bases. The dissolution medium was $500 \mathrm{ml}$ of distilled water maintained at $37 \pm 0.5^{\circ} \mathrm{C}$. The suppository was held in a basket rotating at $50 \mathrm{rpm}$. A sample of $2 \mathrm{ml}$ of the release medium was withdrawn at predetermined time intervals and replaced with an equal volume of fresh release medium. The collected samples were assayed spectrophotometrically at $302 \mathrm{~nm}$. Samples of the release medium of unmedicated suppositories were used as blanks.

\section{Stability testing}

This test was carried out on three formulations with the highest amount of drug release after 2 hours and having acceptable physical characteristics (hardness, melting time and content uniformity). The formulations were: F3, F2 and F3 + 4\% eudispert gel.
Samples of 1 gram weight suppository, each containing $100 \mathrm{mg}$ salicylamide were packed in separate glass containers, protected from light and stored at room temperature for one year. Aliquots were taken and assayed for drug content at the beginning of the experiment and at time intervals of 1, 2, 4, 6, 8, 10 and 12 months.

\section{Validation of assay method for stability study}

The assay method was validated by preparing two known concentrations of salicylamide solutions in distilled water, one was diluted with an equal volume of $1 \mathrm{M}$ sodium hydroxide solution and the other with an equal volume of hydrogen peroxide (20 volume). The two diluted solutions were left to stand for 48 hours, among them were 2 hours in direct sunlight. The two solutions were assayed for drug contents. The decomposition was found to be completed $^{22}$. 


\section{RESULTS AND DISCUSSION}

Two types of suppository bases were used in this study to prepare salicylamide suppository namely emulsifying fatty bases (Witepsol) and water soluble bases (PEG). All the prepared salicylamide suppositories showed acceptable physical characteristics with respect to hardness, melting time and uniformity of drug contents. The hardness ranged from 5.80 to $6.45 \mathrm{~kg}$, melting time from 19 to $35 \mathrm{~min}$ and drug content from 98.44 to $102.13 \%$.

The results of drug release revealed that the release of salicylamide from the investigated formulations was markedly affected by the nature of the base. We can interpret the variations of the release of salicylamide from different suppositories by relying on the physical properties of the used bases as their melting point and hydroxyl values. Hydroxyl value reflects the hydrophilic properties of the base, which in turn can modify both the release and the absorption rates of the drug. The melting point affects the rate of getting the drug free in the release medium. From Figure (1-a), we can see that after $2 \mathrm{hr}, \mathrm{F} 3$ which prepared using witepsol 35, showed the highest drug release value among all the tested formulae $(92.6 \%)$. This base is fatty in nature, has a low melting point $(33.5-35.5)^{\circ} \mathrm{C}$ and a high hydroxyl value $(40-50)$. All these characteristics may enhance the drug release by increasing the hydrophilic environment around the drug and getting it free in the dissolution medium in a short time. Voiget and $\mathrm{Falk}^{23}$ found that the release of drug from fat bases is directly proportional to the solubility of drug in water. These interpretations can be applied also to $\mathrm{F} 2$ and $\mathrm{F} 1$, which contain witepsol W 25 and witepsol $\mathrm{H} 15$ and showed $90.82 \%$ and $80.15 \%$ drug release, respectively.

The release from PEG bases, Figure (1-b) was found to be molecular weight dependent. Increasing the percentage of high molecular weight polymer led to decreasing the amount of drug release and vice versa. This is because the higher the molecular weight of PEG, the higher the melting point.

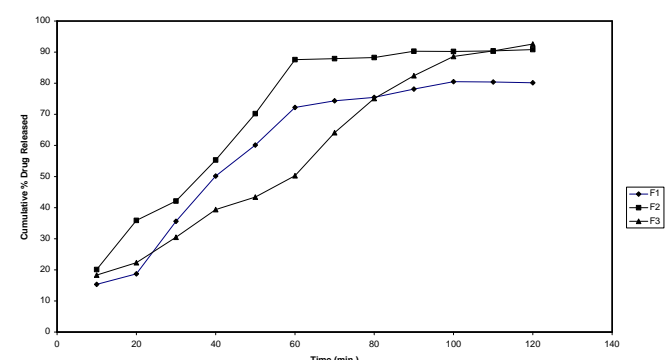

Fig. 1-a: Dissolution profiles of salicylamide from fatty bases in distilled water.

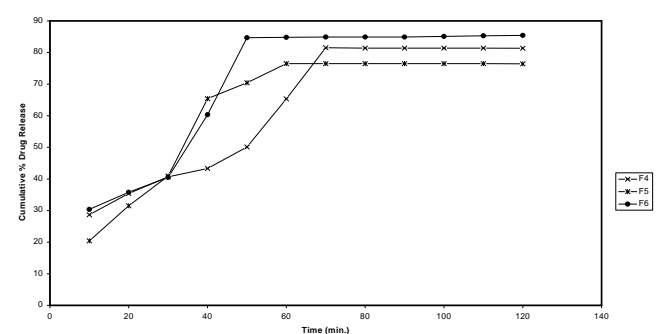

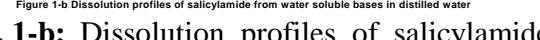
from water soluble bases in distilled water. 
The use of 50\% PEG 6000 in F5 delayed the release of salicylamide from suppositories. Formula no.6 showed the highest amount of drug released among this group because it contains a high percentage $(75 \%)$ of PEG 1000. So, the release of salicylamide from PEG bases could be ranked as F6 > F4 > F5.

Eudispert gel was mixed with suppository bases to enhance the availability of salicylamide. It was observed that eudispert gel affected inversely the hardness and melting time of the prepared suppositories. This effect was directly proportional to the concentration of the gel, yet, the suppository still have acceptable hardness and melting time. Figures (2 and 3) show the effects of different concentrations of eudispert gel on hardness and melting time.

No one rule was found to control the effect of gel on drug content uniformity (Figure 4). The rate and extent of release of salicylamide from suppository base were affected by the addition of gels. In most of these cases, the release of drug was enhanced with the incorporation of low concentration of gel $(2 \%)$ and decreased with $4 \%$ and $6 \%$ gel concentrations. This was observed in formulae F1 and F3. this may be due to the fact that low gel concentration increases the softness and hence drug release. However, increasing the concentration increased the viscosity of the base and hence led to the entrapment of the drug inside. No one rule was found to control the effect of gel on drug content uniformity
(Figure 4). The rate and extent of release of salicylamide from suppository base were affected by the addition of gels. In most of these cases, the release of drug was enhanced with the incorporation of low concentration of gel $(2 \%)$ and decreased with $4 \%$ and $6 \%$ gel concentrations. This was observed in formulae F1 and F3. this may be due to the fact that low gel concentration increases the softness and hence drug release. However, increasing the concentration increased the viscosity of the base and hence led to the entrapment of the drug inside.

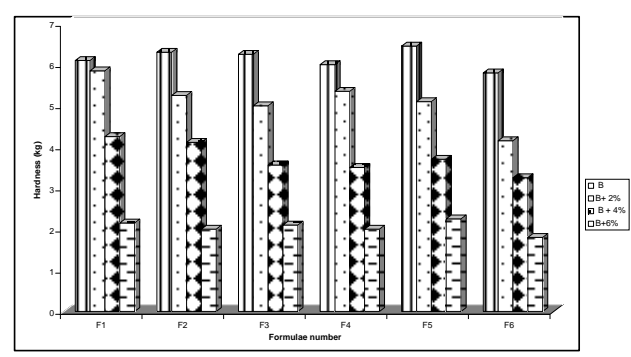

Fig. 2: Effect of eut eudispert hiv hardness of salicylamide suppositories

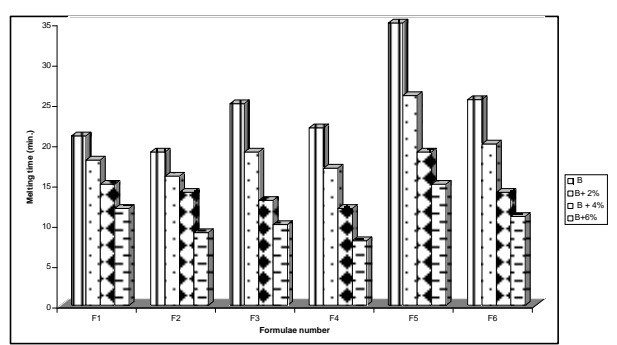

Fig. 3: Effect of eudispert hv gel on melting time of salicylamide suppositories 


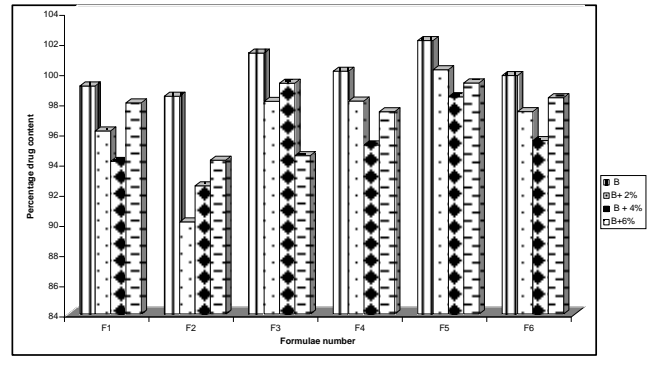

Fig. 4: Effect of eudispert hv gel on content uniformity of salicylamide suppositories

Nixon et al. ${ }^{24}$ and Florence et al. ${ }^{25}$ found that the rate of drug release is inversely proportional to the viscosity of the carrier base.

$2 \%$ eudispert gel increased the drug release in case of F1, F3, F4, F5 and F6. while, on increasing the gel concentration to $4 \%$ and $6 \%$, the percentage drug released was decreased. The drug release from formula F2 was not consistent (Figure $5)$. From these data one can conclude that $2 \%$ eudispert gel is the most accepted concentration to enhance the release of salicylamide from fatty bases, while, $4 \%$ and $6 \%$ increased the viscosity and considerably decreased the release. Regarding PEG bases, it was found that drug release was directly proportional to the concentration of the gel. From the previous results, we can rank the investigated formulations according to their physical characteristics and release profiles (Table 3 ). Therefore, we can select formulations of F3, F2 and $\mathrm{F} 3+4 \%$ eudispert for stability study.
The assay method modified for stability study showed $100 \%$ decomposition of salicylamide solution. This reflects the validity of this method to study the stability of salicylamide suppositories.

Figure (6) shows the remaining salicylamide versus time. The selected suppository formulations showed acceptable stability. The formulae can be arranged according to their stability, descendingly, as follows: F3+4\% eudispert, F3 and F2.

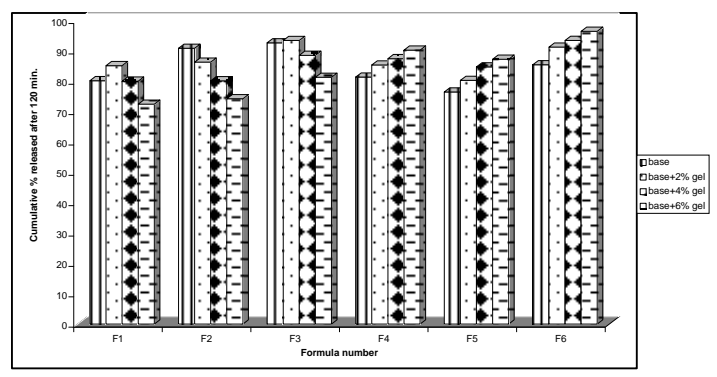

Fig. 5: Effect of eudispert hy gel on

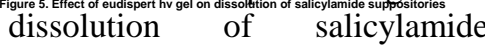
suppositories.

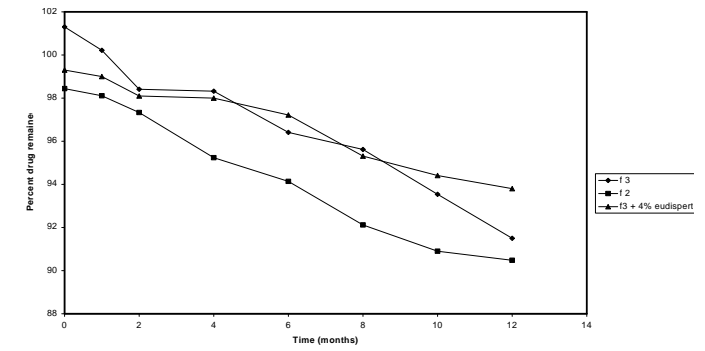

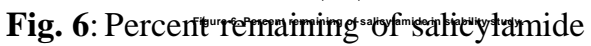
in stability study. 
Table 3: Rank order of the investigated formulations according to their physical characteristics and dissolution profiles.

\begin{tabular}{|c|c|c|c|c|c|}
\hline Formula & Hardness & $\begin{array}{c}\text { Melting } \\
\text { time }\end{array}$ & $\begin{array}{c}\text { Content } \\
\text { uniformity }\end{array}$ & $\begin{array}{c}\text { Dissolution } \\
\text { rate }\end{array}$ & $\begin{array}{c}\text { Total rank } \\
\text { order }\end{array}$ \\
\hline F1 & 4 & 14 & 8 & 19 & 45 \\
F1 + 2\% gel & 6 & 11 & 17 & 14 & 48 \\
F1 + 4\% gel & 12 & 8 & 22 & 21 & 63 \\
F1 +6\% gel & 20 & 5 & 14 & 24 & 63 \\
\hline F2 & 2 & 12 & 9 & 6 & $\underline{29}$ \\
F2 + 2\% gel & 9 & 9 & 24 & 11 & 53 \\
F2 + 4\% gel & 14 & 7 & 23 & 20 & 64 \\
F2 +6\% gel & 22 & 2 & 21 & 23 & 68 \\
\hline F3 & 3 & 16 & 2 & 4 & 25 \\
F3 + 2\% gel & 11 & 12 & 13 & 3 & 39 \\
F3 + 4\% gel & 16 & 6 & 7 & 8 & 37 \\
F3 +6\% gel & 21 & 3 & 20 & 17 & 61 \\
\hline F4 & 5 & 15 & 3 & 16 & 39 \\
F4 + 2\% gel & 8 & 10 & 12 & 13 & 43 \\
F4 + 4\% gel & 17 & 5 & 19 & 9 & 50 \\
F4 +6\% gel & 22 & 1 & 16 & 7 & 46 \\
\hline F5 & 1 & 19 & 1 & 22 & 43 \\
F5 + 2\% gel & 10 & 18 & 4 & 18 & 50 \\
F5 + 4\% gel & 15 & 12 & 10 & 15 & 52 \\
F5 +6\% gel & 19 & 8 & 6 & 10 & 43 \\
\hline F6 & 7 & 17 & 5 & 12 & 41 \\
F6 + 2\% gel & 13 & 13 & 15 & 5 & 46 \\
F6 + 4\% gel & 18 & 7 & 18 & 2 & 45 \\
F6 +6\% gel & 23 & 4 & 11 & 1 & 39 \\
\hline \hline
\end{tabular}

\section{Conclusions}

The prepared salicylamide suppository formulations showed acceptable physical characteristics with respect to hardness, melting time and uniformity of drug content. The amount of drug released from fatty bases is available and it increases with the increase in hydroxyl value of the base and so, F3 which contain Witepsol W35 with low melting point and the highest hydroxyl value showed the highest dissolution rate after $2 \mathrm{hr}$ among the fatty bases, but in case of PEG bases, it was molecular weight dependent.

Addition of 2\% eudispert gel increased the release from all the investigated formulae (except F2), but increasing the gel concentration to $4 \%$ then to $6 \%$ showed different effects on the release.

One year stability study revealed that the selected formulations would result in an acceptable stability profile for remaining salicylamide. Rectal 
suppositories of salicylamide could be prepared to be used as an alternative to the oral dosage form to circumvent the first - pass metabolism and to be used for people with special circumstances.

\section{REFERENCES}

1- M. E. Aulton, The science of dosage form design, $2^{\text {nd }} \mathrm{Ed}$. Leicester, UK, 2002, pp. 534537.

2- J. H. Ewoud, G. B. Albertus, and D. B. Douwe, Clin. Pharmacokinet., 21, 11 (1991)

3- A. G. de Boer, F. Moolenaar, L. G. de Leede, D. D. Breimer, ibid., 7, 285 (1982).

4- J. H. Ewoud, G. B. Albertus and D. B. Douwe, ibid., 21, 110 (1991).

5- M. Jay, R. M. Beihm, G. A. Digenis, F. A. Deland, L. Coldwell and A. R. Mlodozeniec, J. Pharmacol., 37, 266 (1985).

6- L. G. de Leede, A. G. de Boer, C. P. Roozen, and D. D. Breimer, J. Pharmacol. Exp. Ther., 225, 181 (1983).

7- K. Takada, H. Yoshimura, N. Shibata, Y. Masuda, H. Yoshikawa, S. Muranish, T. Yasumura, and T. Oka, J. Pharmacobio-Dyn., 9, 156 (1986).

8- A. G. de Boer, L. G. de Leede, and D. D. Breimer, Br. J. Anaesth., 56, 69 (1984).

9- A. G. de Boer, D. D. Breimer, J. M. Gubbens-Stibbe and A.
Bosma, Biopharm. Drug Dispos., 4, 321 (1983).

10- A. G. de Boer, J. M. GubbensStibbe and D. D. Breimer, J. Pharm. Pharmacol., 33, 50 (1981).

11- G. Levy and T. Matsuzawa, J. Pharmacol. Exp. Ther., 156, 285 (1967).

12- S. Reiegelman and M. Rowland, J. Pharmacokinet. Biopharm., 1, 419 (1973).

13- J. Shibasaki, R. Konishi, M. Koike, A. Imamura and M. J. Sueyasu, Pharmacobio-Dyn., 4, 91 (1981).

14- S. M. Pond, J. A. Waschek, V. Mahacha, R. M. Fielding, D. J. Effeney and T. N. Tozer, J. Pharmacol. Exp. Ther., 245, 291 (1988).

15- "Muscle-Pain Reliever Is Blamed For Staten Island Runner's Death", New York Times, 200706-10.

16- T. Ogiso, M. Twaki, T. Tanino, J. Fujii, and T. Paku, Biol. Pharm. Bull., 16, 315 (1993).

17- H. Umejima, N. S. Kim, T. Ito, T. Uchida and S. Goto, J. Pharm. Sci., 82, 195 (1993).

18- N. S. Kim, M. Kawata, T. Uchida and S. J. Goto, ibid., 81, 537 (1992).

19- N. S. Kim, M. Kawata, T. Uchida and S. Goto, ibid., 81, 904 (1992).

20. Service Bulletin on Eudispert Acryl Resin, Rohm. GMBH Weiterstadt, Germany. 
21. USP XXV (2005), The united States Pharmacopiea, 24, 56, USA.

22- I. T. Ehab, A. Z. Abdel-Azim, A. K. Alaa and A. K. Mansoor, Phamaceutical development and Technology, 2003, Vol. 8, No. 1, pp. 21-30.

23- R. Voigt and G. Falk, Pharmazie, 23, 709 (1968).
24- J. R. Nixon, P. P. Glorgakopuvlos and J. E. Corless, J. Pharm. Pharmacol., 19, 246 (1967).

25- A. T. Florence and D. Attwood, Physicochemical principles of pharmacy, $3^{\text {rd }}$ Ed., Easton and Bristol: London, 1998, pp. 441446. 